\title{
Simulation Spread Spectrum Signal Based on MATLAB
}

\author{
Xingyuan Xu, Lili Xia \& Sheng Liang \\ China Satellite Maritime Tracking and Control Department, Jiangyin, 214431, China
}

ABSTRACT: In this paper, the structure and characteristics of the spread code and spread spectrum telemetry signal is analyzed in detail, the design language MATLAB simulation model based on spread spectrum telemetry signals and verify its correctness, for the study of acquisition and tracking algorithm provides a reliable source.

KEYWORD: Spreading; MATLAB; Gold

\section{INTRODUCTION}

Spread spectrum telemetry system has strong antiinterference, confidentiality, the advantages of multi-objective for monitoring and control, etc. (Vannee D.J.R et al, 2011), and therefore subject to more and more attention and a large number of applications; the same time (Van Dierendonck, 2006), greatly enhance the computer with signal processing capabilities to a general purpose computer the processing platform (Grant P.M et al, 2008), combined with modern signal processing methods, research spread spectrum telemetry signal acquisition and tracking algorithm and its software implementation (Hurd W.Statman, 2007), monitoring and control equipment to improve the versatility and simplify the complexity of monitoring and control equipment, has important practical significance.

\section{GOLD CODE}

Spread Spectrum System in aerospace measurement and control system, spread spectrum signal is achieved by spreading codes, spreading codes on the performance of the whole system plays a decisive role (Agurre $S$ et al, 2009). Anti-jamming system, anti-noise, anti-fading, anti-interception, covert and confidential information, multiple-access communication and synchronization with the capture and spreading codes are all closely related to the design (Natali F.D, 2012).

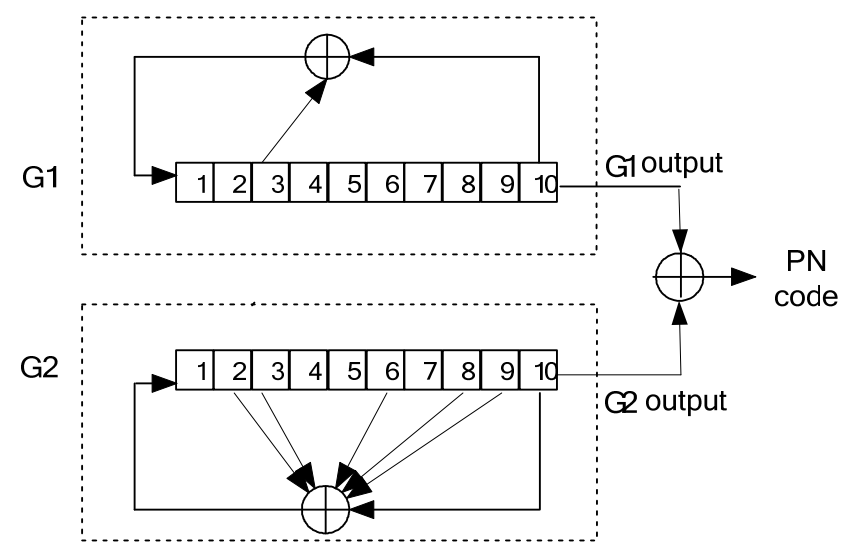

Figure 1. Gold code generator

Gold code sequence with 3-value crosscorrelation function, its value is:

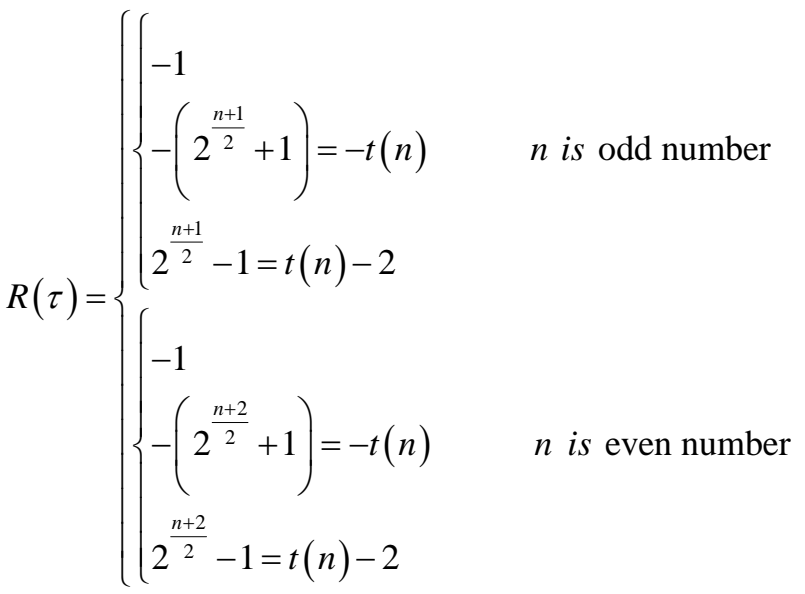

Where,

$t(n)=\left\{\begin{array}{ll}2^{\frac{n+1}{2}}+1 & n=2 k-1 \\ 2^{\frac{n+2}{2}}+1 & n=2 k\end{array} \quad k\right.$ is an integer, but is not a multiple of 2 
Gold code autocorrelation function is a function of three values, but with different frequency of occurrence.

For the case of $n=10$, and only when, the autocorrelation function can only obtain maximum 1023; when other values, the cross-correlation value is -65 , -1 , or 63 . Because of this nature Gold code sequence, the receiver can pass phase shift local pseudo-code to the received signal in the pseudo-code phase alignment to obtain high processing gain, it can be buried in the noise the signal detected.

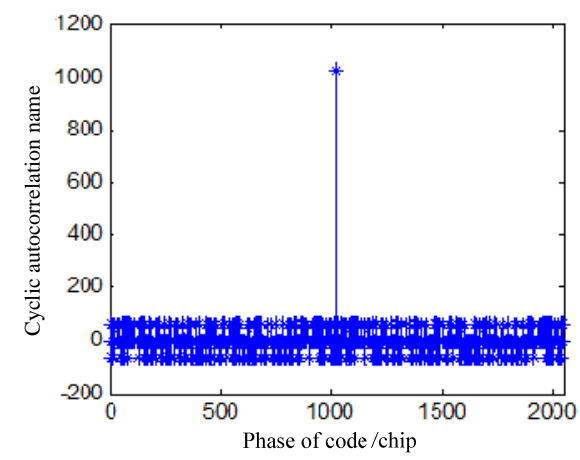

(a). Autocorrelation function

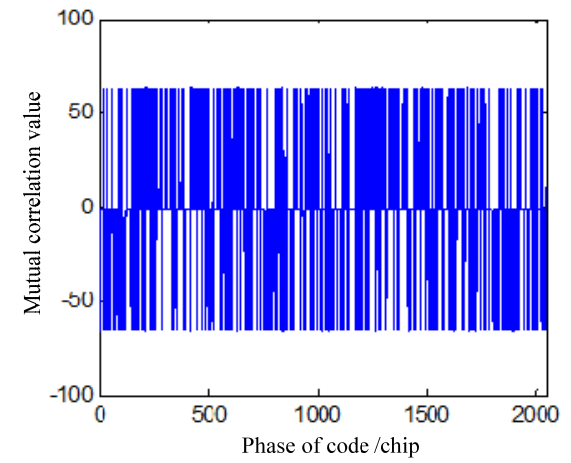

(b). Cross-correlation function
Figure 2 is the autocorrelation function of the code length 1023 Gold code sequence and crosscorrelation functions.

\section{SPREAD SPECTRUM SIGNAL}

Spread-spectrum signal using QPSK modulation in general power imbalance that UQPSK modulation. Modulation principle is shown in Figure 3.

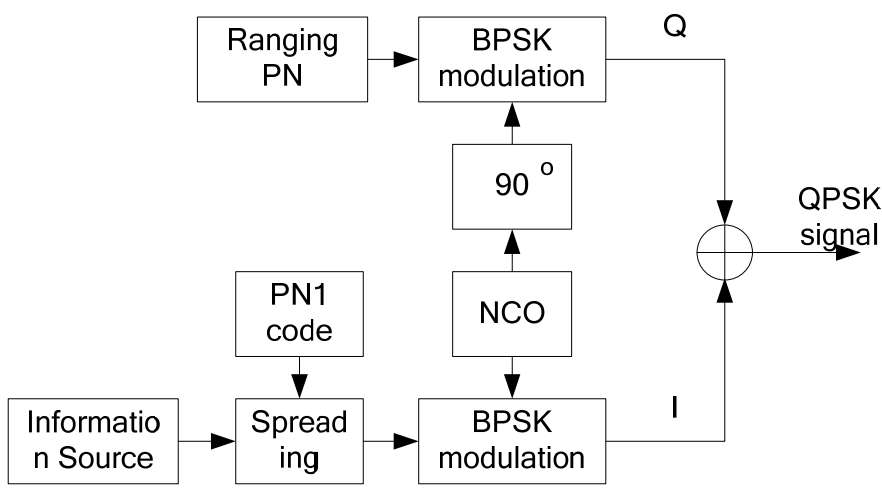

Figure 3. Schematic spread QPSK modulation scheme

Wherein the I branch modulation telemetry information using spreading Gold code of length 1023, $\mathrm{Q}$ modulation branches truncated m-sequence of length 256 as $1023 *$ Ranging code transmission of the data I assign a higher signal power branch . Short code used to guarantee precision ranging, and the long code for defuzzification.

Mathematical expression of the QPSK signal is:

Figure 2. Autocorrelation and cross-correlation function Gold code sequence

$S(t)=\sqrt{2 *(0.91) P_{S}} P N_{I}(t) d(t) \cos (2 \pi f t+\phi)+\sqrt{2 *(0.09) P_{S}} P N_{Q}(t) \sin (2 \pi f t+\phi)$

\section{PRE-MODULATION FILTERING}

In this paper, the pseudo code rate 5.115Mchips/s, bandwidth of $10.23 \mathrm{MHz}$, so shaping filter $3 \mathrm{~dB}$ bandwidth of $5.115 \mathrm{MHz}$, set off with a $6 \mathrm{MHz}$, rolloff factor of 0.45 . The shaping filter 56 Order, its amplitude-frequency characteristic and phasefrequency characteristics shown in Figure 4:
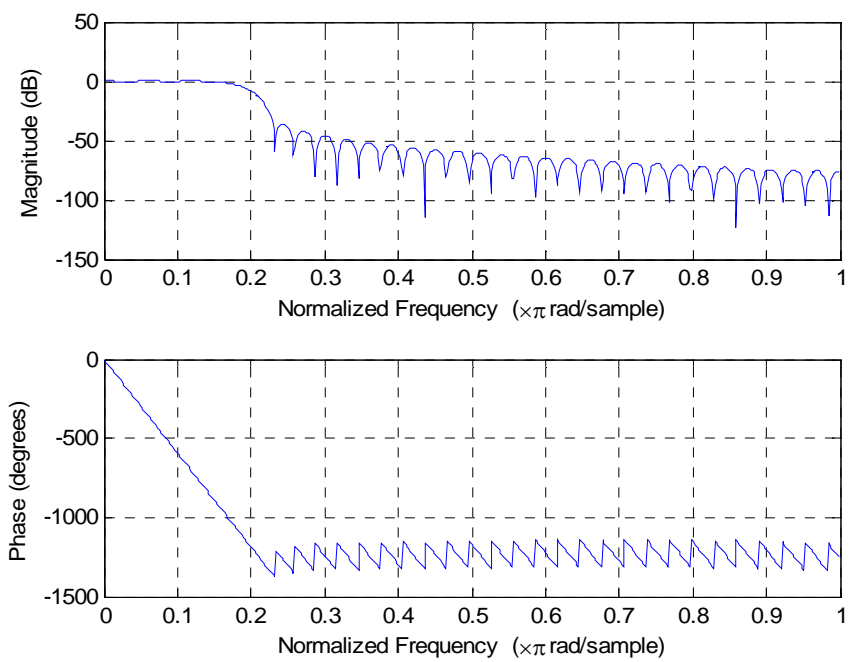

Figure 4. The amplitude-frequency characteristics and phasefrequency characteristics 
Figure 5 respectively after shaping filter after time domain and frequency domain waveforms:
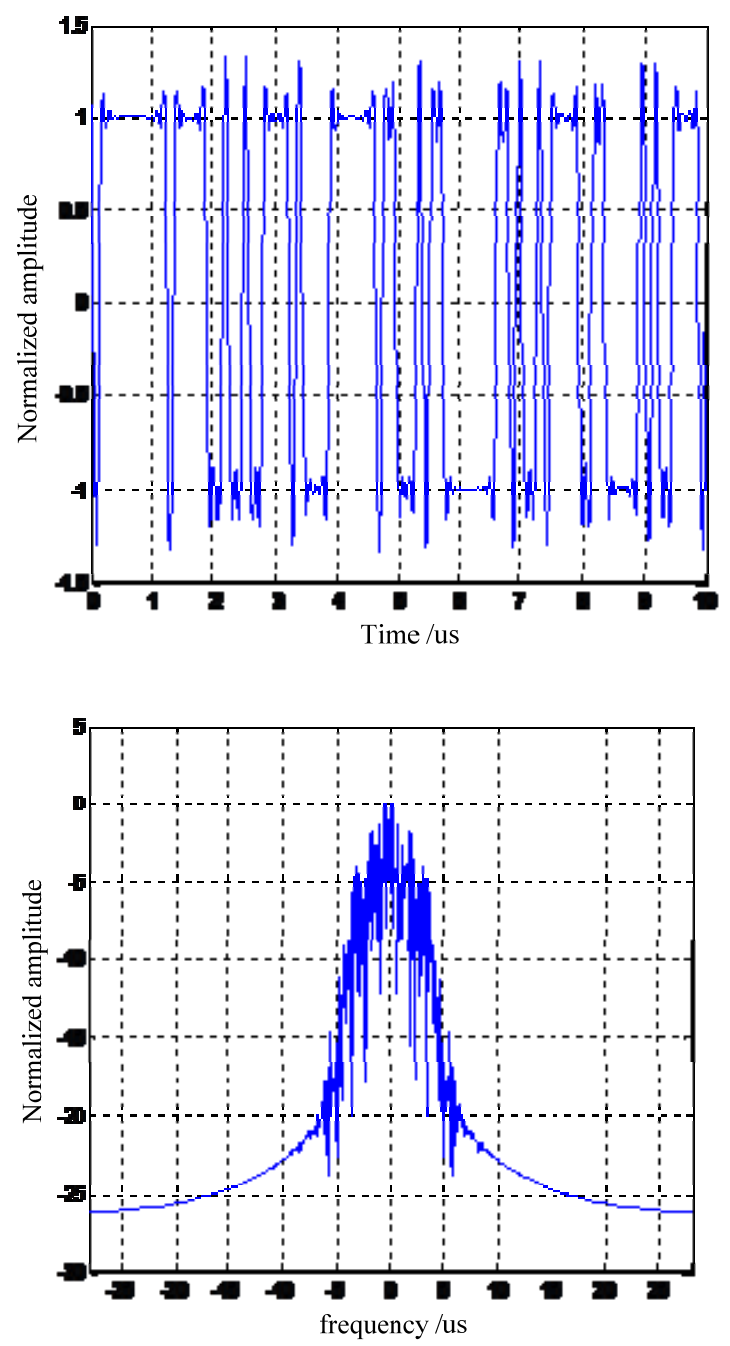

Figure 5. Time domain and frequency domain waveforms after shaping filter

\section{SUMMARY}

This chapter of the spread spectrum telemetry signal characteristics and structure of constructed based on spread spectrum telemetry signal MATLAB simulation model, and its correctness was verified for the study of the spread-spectrum signal acquisition and tracking algorithm of the foundation.

\section{REFERENCES}

Agurre S, Hinedi.S. Two Novel Automotive Frequency Tracking Loops[J]. IEEE Transactions on Aerospace and Electronic System. 2009.25.

Grant P.M, Spangenberg S.M, Scott J.etal. Doppler estimation for fast acquisition in spread spectrum communication systems [J]. IEEE 2008
Hurd W.Statman. High dynamic GPS receiver using maximum likelihood estimation and frequency tracking[J]. IEEE Trans on AES July. 2007.

Natali F.D.AFC Tracking Algorithms[J]. IEEE Trans on COM.Aug 2012.

Van Dierendonck. GPS Receivers in Global Positioning System [J].Theory and Application.Vol.I.2006. 329 407.

Vannee D.J.R, Coenen A.J.R.M. New Fast GPS codeacquisition technique using FFT[J]. Electronics Letters.2011.27:158-161. 